\title{
LA PREVENCIÓN DE CONFLICTOS BÉLICOS EN EL DERECHO INTERNACIONAL ACTUAL. LAS NACIONES UNIDAS Y EL SISTEMA INTERAMERICANO
}

\section{Héctor GROS ESPIELL*}

RESUMEN: El autor hace una revisión de la prevención de conflictos bélicos como elemento para mantener la paz y la seguridad internacionales. En el marco de las Naciones Unidas, del sistema interamericano y de diversos tribunales internacionales, el autor lleva a cabo un análisis exhaustivo de las formas de prevención como son la diplomacia preventiva, las medidas de confianza, y las operaciones para el mantenimiento de la paz, entre otras.

ABSTRACT: The author evaluates armed conflicts prevention as an element to maintain international peace and security. Under the United Nations framework, the inter-American system and severan international tribunals, the author carries out an exhaustive analysis of the forms of conflict prevention as preventive diplomacy, trust measures, and peacekeeping operations, among others.

RÉSUMÉ: Dans le cadre de l'Organisation des Nations Unies, du système Interaméricain et de divers tribunaux internationaux, l'auteur fait un état des lieux de la politique de prévention des conflits armés comme un élément de maintien de la paix et de la sécurité internationale. Cette analyse exhaustive de l'auteur expose les formes de prévention, tels que la diplomatie préventive, les mesures de confiance, les opérations de maintien de la paix, entre autres.

* Profesor de derecho internacional. Miembro de l'Institut de Droit International, del Instituto Hispano Luso Americano de Derecho Internacional y del Consejo del Istituto Internazionale di Studi sui Diritti dell'Uomo (Trieste). 
La prevención de conflictos constituye hoy un elemento esencial, absolutamente necesario, para que pueda cumplirse y tener efectividad el "propósito" primario de las Naciones Unidas de "mantener la paz y la seguridad internacionales" (artículo 1.1 de la Carta de las Naciones Unidas).

Esta idea de que el mantenimiento de la paz y la seguridad internacionales exige no sólo "suprimir actos de agresión y otros quebrantamientos de la paz" sino también prevenirlos para impedir su estallido, no es únicamente una afirmación conceptual y un resultado de la experiencia histórica, sino que ya se encuentra en la Carta de las Naciones Unidas. En efecto en el ya citado artículo 1.1, se establece que "con tal fin" ("Mantener la paz y la seguridad internacionales"), las Naciones Unidas deben "tomar medidas colectivas eficaces para prevenir y eliminar amenazas a la paz”.

La idea de que la prevención de los conflictos constituye un aspecto esencial para lograr el propósito de asegurar y mantener la paz y la seguridad internacionales, ya fue consagrada jurídica y políticamente en la carta. ${ }^{1}$ La exégesis de la carta permite distinguir entre la prevención para mantener la paz y las acciones a adoptar luego de que la paz se ha roto, para restaurarla y reestablecerla. La experiencia histórica cumplida de 1946 hasta hoy no ha hecho sino confirmar este enfoque, agregando ahora las medidas posteriores de consolidación de la paz. Estas medidas, al estar dirigidas a impedir el renacimiento de conflictos respecto de los cuales se aplicarían medidas de restablecimiento de la paz, constituyen, a su vez, formas de prevención.

La prevención, siempre necesaria, es además, menos costosa, menos traumática y produce consecuencias políticas y económicas menos graves y menos hirientes, que las medidas que es necesario aplicar para restablecer la paz y la seguridad, una vez que el conflicto ha estallado.

1 La doctrina, durante años, no analizó especialmente el sentido, el alcance y la proyección de la referencia que hace el artículo 1.1 a "prevenir y eliminar amenazas a la paz". Goodrich y Hambro, Commentaire de la Charte des Nations Unies, 1946, Genève, pp. 120 y 121; Jiménez de Aréchaga, Eduardo, Derecho constitucional de las Naciones Unidas, Madrid, 1958, p. 36. Una notable excepción es la de Hans Kelsen, quien con sin igual precisión distinguió entre el "mantenimiento de la paz" por medio de la prevención de conflictos bélicos, del "reestablecimiento de la paz", porque cuando la paz ya ha sido rota, no puede ser mantenida sino restaurada. Kelsen, Hans, The Law of the United Nations, London, Stevens \& Sons, 1951, p. 13. 
La Carta de las Naciones Unidas ha proyectado la idea de la necesaria prevención de los conflictos, en las competencias atribuidas al Consejo de Seguridad, a la Asamblea General y al secretario general (artículos $11.3,12,14,24.2$ y 99 ).

Es cierto que estas normas no se refieren expresamente a la prevención ni a la forma de prevenir los conflictos. Pero al referirse a "situaciones susceptible de poner en peligro la paz y la seguridad internacionales" (artículo 11.3), al establecer que se deberá actuar "de acuerdo con los Propósitos y Principios de las Naciones Unidas" (artículo 24.2) y al señalar el caso de los asuntos que pueden "poner en peligro el mantenimiento de la paz y la seguridad internacionales" (artículo 99), con referencia a competencias de la Asamblea General, del Consejo de Seguridad y del secretario general, la carta está determinando competencias de tipo preventivo de estos órganos, respecto de conflictos que pueden llegar a estallar bélicamente y que, por su mera existencia, ya constituyen en si mismos, y más aún potencialmente, una amenaza para la paz y la seguridad internacionales.

La prevención, supone la posibilidad - y el deber- de encarar la solución de los conflictos por el arreglo pacífico (Carta de las Naciones Unidas, artículo 2.3 y capítulo VI, artículos 33 y 38; y Declaración sobre los Principios de Derecho Internacional referentes a las Relaciones de Amistad y Cooperación entre los Estados (Resolución 2625XXV) y por la acción en caso de "toda amenaza a la paz" (capítulo VII, artículo 39 de la carta). En uno u otro caso, ya sea por la vía de la solución pacífica o de la acción "preventiva" (artículo 50), se trata de formas de prevención de conflictos bélicos.

Hay que destacar que con respecto al "principio" de interdicción del uso de la fuerza", el artículo 2.4 de la carta impone la abstención de la utilización de la misma y también de la "amenaza" del uso de la fuerza (Declaración sobre los Principios de Derecho Internacional Referentes a las Relaciones de Amistad y Cooperación entre los Estados) (El principio de que los Estados en sus relaciones internacionales se abstendrán de recurrir a la amenaza o uso de la fuerza).

La amenaza del uso de la fuerza es, en consecuencia, uno de los elementos que puede llevar a la adopción de medidas de prevención de los conflictos, antes de que haga efecto el uso de la fuerza. 
Es interesante destacar que el artículo 50 de la carta, retomando la terminología del artículo 1.1 ("prevenir y eliminar amenazas a la paz"), se refiere a las "medidas preventivas".

El artículo 52 de la carta (capítulo VIII. Acuerdos regionales), al establecer que "Ninguna disposición de la carta se opone a la existencia de acuerdos u organismos regionales cuyo fin sea entender en los asuntos relativos al mantenimiento de la paz y la seguridad internacionales y susceptibles de acción regional", está aceptando que estos acuerdos u organismos pueden actuar para prevenir conflictos regionales. Esta acción regional, de carácter previsional, no implicaría naturalmente la aplicación de "medidas coercitivas" sin autorización del Consejo de Seguridad (artículo 53).

El concepto de lo que es la prevención de los conflictos bélicos - prevención que no sólo es un deber, sino que como hemos ya dicho es necesaria y constituye una esencial contribución al mantenimiento de la paz-, es algo totalmente distinto a la que se ha llamado legítima defensa preventiva.

La legítima defensa sólo se configura - pese a ser un derecho inmanente de los Estados, de acuerdo con lo establecido en el artículo 51 de la Carta de las Naciones Unidas-, en caso "de ataque armado". No puede invocarse el derecho de legítima defensa, individual o colectivo, para prevenir un posible ataque armado que aún no se ha hecho efectivo. En una situación de peligro de un ataque armado previsible o posible, sólo cabe poner el hecho y la situación en conocimiento del Consejo de Seguridad para que éste tome de inmediato todas las medidas requeridas para mantener la paz y la seguridad internacionales.

Aunque la solución que sostengo puede ser discutible y ha dividido a la doctrina, pienso hoy, y me reafirmo en el criterio de que en aras de la paz y la seguridad no puede aceptarse la llamada legítima defensa preventiva. Incluso los que se resisten a aceptar de manera total la afirmación de la ilicitud de la legítima defensa preventiva, reconocen la enorme peligrosidad de su admisión. ${ }^{2}$

2 Pendant les premières années du XXI siècle, l'Union Européenne connaîtra un processus de croissance sans précédent. De l'actuelle Europe des quinze elle passera à 27 ou 28 membres. L'entrée 
Creo que lo que hay que afirmar, jurídica y políticamente, es que no hay un derecho de legítima defensa preventiva y que conceptualmente la prevención de los conflictos bélicos no puede realizarse mediante el uso de la fuerza, invocando la legítima defensa.

\section{III}

El mantenimiento de la paz — que obliga a la adopción de medidas de prevención de los conflictos por aplicar antes de que éstos estallen- supone para su eficacia un sistema de información, de previsión y de reacción urgente, adecuada, justa, equilibrada, y no discriminatoria. Esto ha sido señalado por los sucesivos secretarios generales de las Naciones Unidas, en los documentos a los que luego nos referiremos especialmente. Algo se ha hecho, pero es mucho más lo que resta por hacer. ${ }^{3}$

El mantenimiento de la paz (peace keeping), debe ser paralelo con la construcción de la paz (peace building), ${ }^{4}$ concepto más amplio y global, que abarca la prevención de los conflictos, pero también la lucha por eliminar las causas (políticas, económicas, sociales y culturales), de la violencia y de la confrontación, las hondas y profundas raíces de los conflictos bélicos.

La prevención de conflictos bélicos debe ser adecuada en el tiempo, rápida, inmediata y oportuna. Si no lo es, el conflicto puede estallar y habría que tratar de reestablecer la paz, paz ya rota, en un conflicto bélico ya producido.

Por eso en el caso de la prevención es plenamente aplicable la afirmación de que "Mañana siempre es tarde". 5

des nouveaux pays constitue un défi et cela suppose la prise en compte des diverses cultures et systèmes sociaux structurels très différents de ceux de l'Europe occidentale. Les mécanismes juridiques, politiques et financiers de préparation à l'adhésion sont en marche. L'adaptation au patrimoine communautaire, la cohésion économique et sociale se trouvent parmi les éléments les plus importants pour réussir l'intégration.

3 Mayor Zaragoza, Federico, Un mundo nuevo, Círculo de Lectores, Ediciones UNESCO, 2000, p. 533; Brgacao, Clovis, "Prevenir, mantener e Construir a Paz: Novos Desafios a Segurança International”, O Brasil e as Novas Dimensòes de Segurança International, São Paulo, 1999.

4 Mayor Zaragoza, Federico, Mañana siempre es tarde, Madrid, Espasa Calpe, 1987.

5 Ibidem, p. 6 bis. Sobre la "diplomacia representativa", respecto de la que hay una abundante bibliografía, véase especialmente Boutros-Ghali, Boutros, Agenda pour la Paix, Nueva York, Nations Unies, 1992; y Sipri-Unesco, Peace, Security and Conflict Prevention, Oxford University Press, 1998 , pp. 38 y 143. 
La prevención de los conflictos bélicos internacionales obliga a considerar y actuar, por las Naciones Unidas y en lo pertinente por los organismos y acuerdos regionales - dentro de sus competencias y con respeto del derecho internacional-, ante conflictos bélicos internos que puede tener una proyección internacional, transformándose así en conflictos bélicos internacionales.

La prevención de los conflictos debe ser global y totalizante. Si no posee este carácter será siempre ineficaz e incompleta.

\section{IV}

La Carta de la Organización de Estados Americanos —es decir del sistema regional americano, que constituye un acuerdo y un organismo regional según la Carta de las Naciones Unidas-, no contiene normas especiales sobre la forma de ejercer la prevención de conflictos bélicos regionales.

Pero la remisión expresa a los "propósitos" de las Naciones Unidas (Preámbulo, párrafo sexto), y su propio propósito de afianzar la paz y la seguridad del Continente" (artículo 2,a), de "prevenir las posibles causas de dificultades y asegurar la solución pacífica de controversias que surjan entre los Estados miembros (artículo 2.c), muestra que la prevención de los conflictos bélicos está entre las acciones que la OEA —en cuanto acuerdo y organismo regional-, puede y debe desarrollar.

Esta prevención se ejercerá por medio de la solución pacífica de controversias (capítulo V, artículos 2326) o por la aplicación del sistema de "seguridad colectiva" (capítulo VI), en virtud del cual se podrán aplicar "medidas y procedimientos", entre otros casos, ante "cualquier hecho o situación que puede poner en peligro la paz de América", desarrollando así "los principios de la solidaridad continental" (artículo 28).

Al referirse a "cualquier hecho o situación que pueda poner en peligro la paz de América" (artículo 28), la Carta de la OEA encara la posible acción ante eventualidades que pueden exigir la adopción de medidas $o$ acciones preventivas.

Será el Consejo de la Organización (capítulo XII) el que —sin perjuicio de las Competencias de la Asamblea General (artículo 53)—, deberá actuar con respecto a la adopción de las acciones o medidas preventivas (artículos 81-90). 
Con el correr de los años, la conciencia de la importancia determinante de la prevención de los conflictos bélicos, ha permitido tanto a nivel universal como regional construir, sobre las bases normativas antes citadas, todo un sistema conceptual relativo a la prevención de los conflictos bélicos, los medios a utilizar y la estrategia a seguir. Incluso se ha ido precisando lo relativo a la "diplomacia preventiva", especialmente multilateral, ya sea en el ámbito de las Naciones Unidas o de la OEA -y en ocasiones de la acción conjunta y coordinada de ambos- como un ejercicio cada día más necesario.

\section{VI}

Es imposible en este breve trabajo citar la larga enumeración de documentos de las Naciones Unidas que han ido construyendo en el derecho internacional y en la práctica de la vida internacional, el concepto de prevención de conflictos bélicos en su relación con el mantenimiento de la paz. ${ }^{6}$

Citaremos, por tanto, sólo dos textos, a los que atribuimos singular importancia. Uno es la Introducción a la Memoria del Sistema General sobre la Labor de la Organización (1991). En este documento, Javier Pérez de Cuéllar afirmó:

6 Esta enumeración puede encontrarse en la abundantísima bibliografía especialmente anglosajona, que existe al respecto. Entre ellas cabe destacar: Chopra, Jarat y Weiss, Thomas G., Preventing Conflict in the Post-Comunist World: Mobilizing International and Regional Organizations, Washington, D. C., Brookings Press, 1996; Lound, Michael, Preventing Violent Conflicts: A Strategy for Preventive Diplomacy, Washington D. C., United States, Institute for Peace Press, 1995; Dixon, William J., Third-Party Techniques for Preventing Conflict Escalation and Promoting Peaceful Settlemente International Organization, Autumn, 1996; Stedman, Stephen John, "Alchemy for a New World Order: Overselling 'Preventive Diplomacy'", Foreign Affairs, mayo-junio de 1995; Lund, Michael S., "Underrating Preventive Diplomacy", Foreign Affairs, julio-agosto de 1995; Cahill, Kevin M., Preventive Diplomacy: stopping wars before they start, Nueva York, Routledge, Center for International Health and Cooperation, 2000; Zartman, William, Preventive negotiation, avoiding conflict escalation, Lanham, Rowman \& Littlefield Publishers, 2001; Cohen, J., Conflicts Prevention in the OSCE: an assessment of capacities, The Hague, Netherlands Institute of International Relations "Clingendeal", 1999; Carnegie Commission on Preventing Deadly Conflict, Preventing Deadly Conflict: Final Report, Nueva York, diciembre de 1997; Conflict prevention, G8 Foreign Ministers Meeting, Miyazaki, Japón, julio de 2000. 
Una labor de esta naturaleza parece indispensable si queremos desarrollar la capacidad preventiva a que puede recurrir el Secretario General. La falta perenne de este tipo de capacidad entorpece la aplicación del Artículo 99 de la Carta, sobre todo en su aspecto de anticipación. En la Carta no se prevé que las Naciones Unidas deben esperar a que se desencadenen las hostilidades, se produzca una agresión o sean desproporcionadas las violaciones de los derechos humanos antes de tomar medidas para rectificar la situación. Son demasiados los casos en que, en situaciones en que se cernía la amenaza de conflictos en gran escala, se ha mantenido en reserva la capacidad de mediación o de investigación de la Organización, en tanto que se desencadenaban guerras y se enconaban las controversias. Hay complementariedad en que el Secretario General disponga de todos los medios que presupone el Artículo 99 de la Carta y el Consejo de Seguridad (de conformidad con el espíritu del Artículo 34) mantenga un temario de paz que no se limite únicamente a los temas inscriptos oficialmente a solicitud del Estado o los Estados interesados. A mi juicio, esta complementariedad puede hacer que la diplomacia preventiva, de ser una frase, pase a ser una realidad práctica.

La contención o solución de los conflictos está comprendida en el arreglo pacífico de controversias — al cual la Carta dedica todo un capítulo-, pero no es sinónimo exacto de él. La hipótesis básica del capítulo VI — que repito para hacer hincapié en ella- es que ni el propio Consejo de Seguridad ni los Estados Miembros de las Naciones Unidas pueden quedarse cruzados de brazos mientras una situación de fricción desemboca en actos de beligerancia. No obstante, el requisito primordial para que funcionen los mecanismos de arreglo es que cambie radicalmente la opinión que merecen a las parte en las grandes controversias internacionales la función y la capacidad de las Naciones Unidas, y no sólo su órgano judicial, son una tribuna de litigios que en cada caso probablemente emitirá un veredicto negativo para una u otra parte. Creo que debemos empezar a promover activamente el concepto de que, salvo en los casos en que se adopten medidas respeto de quebrantamiento de la paz o actos de agresión (cuestiones de las que se ocupa capítulo VII), las Naciones Unidas son, más bien, un instrumento de mediación que puede contribuir a conciliar reclamaciones e intereses legítimos y a alcanzar soluciones justas y honorables. ${ }^{7}$

Un año después, en 1992, el siguiente secretario general, en aplicación de la reunión cumbre en el Consejo de Seguridad, celebrada el 31 de enero del 92, preparó un documento, titulado Agenda pour la Paix, en el

7 Pérez de Cuéllar, Javier, "Introducción", Memoria del secretario general sobre la Labor de la Organización, Nueva York, Naciones Unidas, 1991, pp. 10 y 11. 
que dedica, inteligentemente, varias páginas al análisis de la "diplomacia preventiva" y al "mantenimiento de la paz".

Dice así en algunos de los párrafos incluidos en esas páginas:

le Conseil de sécurité est devenu un instrument central dans la prévention et le règlement des sconflits aussi bien que dans la préservation de la paix. Nos buts doivent être désormais les suivantes:

D'essayer de déceler aussi tôt que possible les situations porteusses de conflit et de parer au danger, par la voie diplomatiquie, avant que la violence ne se déclare.

Au sens le plus large, enfin, d'essayer, d'extirper les causes les plus profondes du conflit; misère économique, injustice sociale et oppression politique. Une morale nouvelle se discerne dès à présent. Que partagent de plus en plus largement les nations et les peuples du monde et qui trouve à s'exprimer dans les dispositions du droit international, dont un bon nombre son le fruit des efforts de notre Organisation.

La diplomatie prèventive a pour objet d'éviter que des différends ne surgissent entre les parties, d'empêcher qu'un différend existant ne se transforme en conflit ouvert et, si un conflit éclate,de faire en sorte quíl s'etende le moins possible.

La diplomatie préventive vise à régler les conflits avant que la violence n'éclate; le rétablissement et le maintien de la paix ont pour objet de metrre fin aux conflits et de préserver la pais une fois qu'elle a été instiurée. En cas de succès, l'une et les autres débouchert sur la consolidation de la paix aprés les conflits, contribuant ainsi á empêcher que les actes de violence ne reprennent entre les nations et les peuples. ${ }^{8}$

\section{VII}

La existencia de una justicia internacional en general y de una justicia penal de carácter individual, también internacional, constituyen elementos que no pueden olvidarse en la prevención de los conflictos bélicos.

Con respecto a la justicia internacional, en especial en el caso de la Corte Internacional de Justicia, esta contribución a la prevención de conflictos es muy importante. En la competencia contenciosa, aunque la corte actúa respecto de casos ya producidos, a posteriori de los hechos, el aporte puede ser significativo en cuanto la solución judicial del diferendo puede significar impedir su desviación hacia fórmulas bélicas para encararlo. 
Lo mismo puede decirse de lo que, en vía contenciosa, puede resultar de la acción de otras jurisdicciones internacionales y, en especial, de la actuación de tribunales arbitrales.

La historia muestra y prueba que la existencia de fórmulas de solución pacífica de controversias por vía judicial o arbitral ha significado positivamente como instrumento o manera de evitar o prevenir el estallido de conflictos bélicos.

Pero además hay que señalar que la Corte Internacional de Justicia, en el ejercicio de su competencia consultiva, puede acrecentar esta contribución a la prevención de conflictos. En efecto, a diferencia de la contenciosa, la competencia consultiva no tiene necesariamente que referirse a situaciones ya ocurridas, sino a cuestiones jurídicas interpretativas que pueden constituir asuntos previos a eventuales conflictos. Y la opinión consultiva de la corte, al delimitar jurídicamente el asunto y fijar el criterio jurídico aplicable, puede constituir una forma eficaz de prevenir un posible conflicto bélico futuro. Ello ha ocurrido, felizmente así, en varias ocasiones en el pasado. ${ }^{9}$

Los tribunales internacionales, como los actuales relativos a la ex-Yugoslavia y Ruanda, pero especialmente el futuro Tribunal Penal Internacional de Roma, pueden ejercer una importante labor en la prevención de conflictos bélicos. El procesamiento y castigo de los responsables de los delitos, especialmente de los crímenes contra la humanidad, contra la paz

9 El presidente de la Corte Internacional de Justicia, señor Mohamed Bedjaoui, ha tratado este asunto, con especial fineza en los discursos pronunciados ante la Asamblea General de las Naciones Unidas en la 49 sesión (13 de octubre de 1994) y 51 sesión (15 de octubre de 1995). En 1994 dijo: "The consideration I have just advanced with essential reference to the contentious procedure before the Court apply, mutatis mutandis, to the advisory procedure, the importance of which has long underestimated. Only of late has there been a real awareness of the potential impact that the Advisory Opinions of the Court can, whether directly or indirectly, have upon the maintenance of peace. A relevant legal question put in a timely manner to the Court may, by the answer it elicits, or indeed of itself, prove to be an effective tool of preventive diplomacy or contribute, even substantially, to the settlement of a dispute that has already arisen. Many an Advisory Opinion has, in the past, had diplomatic and political implications that were by no means inconsiderable". Y en 1996 agregó: "A moment ago, I referred to another trait of the judicial function as characteristic as it is constant. It is the function of courts to heal rather than prevent: contrary to the legislator or the executive, the decisions by which is commonly termed an "existing and pending interest". From this angle, the function of courts is more to "restore" than to "maintain peace"; the way they function is rendered all the more delicate because, as is the case in international society, this function does not form part of a structure with an operational machinery. In this respect, the wholly unique nature of the advisory proceedings before the International Court of Justice, whose "preventive" virtues no longer need to be demonstrated, must be stressed once again". 
y los vinculados con la agresión, puede tener un efecto disuasorio muy importante en la prevención de los conflictos bélicos.

\section{VIII}

Un interesante ejemplo de la aplicación de la prevención de conflictos, que aunque estuvo referido a las relaciones soviético-estadounidenses, tenía una proyección general por la materia tratada y por el significado universal de las relaciones internacionales de las dos superpotencias, fue el Acuerdo para la Prevención de la Guerra Nuclear (PNW, Prevention of Nuclear War), que se comenzó a negociar en 1973, durante la visita de Nixon a Moscú y se concreto poco después durante la visita de Breznev a Washington.

El PNW fue la expresión más lograda de la política de "detente" de esos días. Aunque interpretado en forma distinta por Moscú y por Washington, ${ }^{10}$ hoy debe recordarse como la más importante expresión de la necesidad de encarar la prevención ante el más grave conflicto al que la humanidad puede verse enfrentada: el conflicto bélico nuclear.

Pese a que se señaló que el principal defecto de este acuerdo era que no contenía medidas prácticas viables para poner en aplicación sus disposiciones, ${ }^{11}$ constituye un antecedente esencial, que podría servir de fuente para otros análogos, referidos a los otros tipos posibles de confrontaciones bélicas, que tuvieran en cuenta la experiencia y las enseñanzas políticas de los últimos treinta años.

Marcó un posible camino a seguir que, lamentablemente, no se transitó. Pero la idea puede no estar muerta, y bajo nuevas circunstancias, condiciones, membresía y alcance, puede renacer.

\section{IX}

Evidentemente la manera de encarar la prevención de conflictos bélicos puede seguir diversas vías y formas, entre ellas las de la diplomacia preventiva de las Naciones Unidas, las acciones de diversa naturaleza,

10 Dobrynin, Anatoly, En confianza (1962-1986), México, Fondo de Cultura Económica, 1998, pp. 287-292; Kissinger, Henry, Memorias, t. II: 1973-1977, que cubre el periodo posterior a enero de 1973, en que ocupó la Secretaría de Estado, lapso durante el que se negoció y firmó el acuerdo.

11 Dobrynin, Anatoly, op. cit., nota anterior, p. 292. 
adoptadas por los órganos competentes de la organización, teniendo en cuenta los lineamientos conceptuales dados, entre otros, por los dos secretarios generales antes citados y por los aportes que ha hecho el actual secretario general Kofi Anan. ${ }^{12}$

Una de estas formas, ya ensayada, es la constituida por las llamadas medidas de confianza ${ }^{13}$ - que han tenido un gran desarrollo a nivel universal y regional, en especial luego de la atención que les prestó, en 1975, la Conferencia de Helsinky sobre Seguridad y Cooperación en Europa, que las encaró en su acta final-, que pueden tomar formas distintas de muy diferente intensidad y extensión, como por ejemplo la notificación de la fecha y características de maniobras militares, la observación recíproca, la información y el control de los procesos armamentistas.

A esto hay que agregar la importancia creciente de las operaciones de mantenimiento de la paz por fuerzas militares suministradas por diversos países, que actúan por las Naciones Unidas, previa decisión de sus órganos competentes, y que en múltiples lugares del mundo, en zonas potencialmente explosivas y en las que pueden estallar conflictos bélicos - han constituido en los últimos años - bajo formas distintas, maneras diversas, útiles y necesarias, de prevención de conflictos bélicos. ${ }^{14}$

La relación de la acción militar de las Naciones Unidas con las operaciones para el mantenimiento de la paz, y con el respeto y aplicación del derecho internacional humanitario, constituye un tema de gran importancia e interés actual, unido conceptualmente con la cuestión de la prevención de los conflictos. ${ }^{15}$

12 Anan, Kofi, Towards a Culture of Prevention, Nueva York, Carnegie Commission of Preventing Deadly Conflicts, 1999.

13 Palma, Hugo, Medidas de confianza recíproca, Santiago de Chile, Comisión Sudamericana de Paz, 1998; id., Confianza, desarme y relaciones internacionales, Lima, CEPEI, 1991; id., Seguridad, desarme y cooperación internacional, Lima, Prat Editor, 1992; "Capítulo III. Fomento de la confianza", Medidas de Confianza en Europa y en América Latina, Instituto de Relaciones Europeas Latino Americanas (IRELA), informe 7/95; Sobre Medidas de Confianza Mutua, Documentos de Base, IRELA, octubre de 1995.

14 La bibliografía sobre las operaciones de mantenimiento de la paz es enorme. Una buena reseña se encuentra en Balladoud, Jacqies, L'ONUE et les opérations de maintien de la Paix, París, Pedone, 1971; Quoc Dinh, Ngurgen et al., Droit International Public, 5a. ed, París, LGDJ, 1994, pp. 939 y 940; y Verhoeven, Joe, Droit International Public, Larcier, Bruxelles, 2000, pp. 799-802. Un ejemplo es el caso de Uruguay, cuyas fuerzas armadas tienen una larga tradición en misiones de paz. Véase al respecto El ejército uruguayo en misiones de paz, 1935-1993, Montevideo, Ejército Nacional, 1993. Otro es el de Brasil: véase Perazizo Lanner, Veisser Lisboa, "O Brasil e as Operaçoes de Manutençao de Paz", en varios autores, O Brasil e as Novas Dimmensoes de Segurança International, São Paulo, 1999.

15 Véanse los estudios de Wahlgren, Vieira de Mello, Courten, Shraga, Pfaner y Geiger sobre 
Es también importante considerar — lo que se hará a continuaciónlas operaciones de las Naciones Unidas a este respecto en cooperación con organismos o acuerdos regionales, cuando ello es posible.

Naturalmente, además, toda la enorme gama de medidas en materia de desarme y de limitación armamentista, así como la proscripción de armas nucleares, la creación de zonas libres de armas nucleares o de zonas de paz o la prohibición de ciertos tipos de armas, como por ejemplo las químicas, bacteriológicas y de destrucción masiva, ${ }^{16}$ constituyen formas latu sensu de prevención de conflictos.

Finalmente no puede dejarse de considerar la prevención de conflictos en relación con el fenómeno de la "destructuración" del Estado y del caos consiguiente, como factor de crisis y generador de violencia bélica, tema que la doctrina ha comenzado a analizar. El caso de Somalia es un ejemplo, entre otros, que no puede dejar de evocarse a este respecto. ${ }^{17}$

Veamos ahora esta prevención en el ámbito regional americano. Ya hemos señalado precedentemente el papel, lícito, posible y positivo que los acuerdos u organismos regionales pueden jugar — por múltiples y diversos medios y formas-, en materia de prevención de conflictos bélicos.

Es pertinente recordar lo que dijeron los dos secretarios generales antes citados. Javier Pérez de Cuéllar ha expresado:

A este respecto debo destacar que, en esencia, las Naciones Unidas no se idearon para monopolizar el proceso de paz. En el párrafo 1 del Artículo 33 y en el párrafo 2 del Artículo 52 de la Carta se reconoce expresamente la

esta relación en Comité Internacional de la Cruz Roja, Simposio sobre Acción Humanitaria y Operaciones de Mantenimiento de la Paz, Informe, 22-24 de junio de 1994, Ginebra, 1995; Ryniker, Anne, "Respeto del Derecho Internacional Humanitario por las Fuerzas de las Naciones Unidas", Revista Internacional de la Cruz Roja, núm. 152, diciembre de 1999, pp. 255-264, y sobre todo el informe del secretario general de las Naciones Unidas del 6 de agosto de 1999.

16 La relación del desarme con la prevención de conflictos bélicos ha sido destacada por toda la doctrina correspondiente. Yo mismo la he señalado expresamente en los trabajos citados en la nota 18. Véase, además, Lafer, Celso, As Novas Dimensioes do Desarmamento: os Regimenes de Controle das Armas de Destruçao en Massa e as Perspetivas para a Eliminaçao das Armas Nucleares, s. 1., s. e., s. a.

17 Véase el excelente estudio: Thurer, Daniel, "El Estado desestructurado y el derecho internacional”, Revista Internacional de la Cruz Roja, núm. 152, diciembre de 1999, pp. 193-221. 
función de los organismos o acuerdos regionales en el arreglo pacífico de controversias. En tanto se esté desarrollando un proceso de paz digno de crédito conforme a lo previsto en esos dos Artículos, no habrá motivo alguno para quejarse de que se ha dejado de lado a las Naciones Unidas. No obstante, si no se ha iniciado un proceso de este tipo o bien parece haberse suspendido indefinidamente o haber fracasado rotundamente, no hay mayores motivos para seguir evitando recurrir a las Naciones Unidas. El reconocimiento de al función central de las Naciones Unidas en el sistema internacional no debe ser puramente teórico.

En este contexto es también importante recordar que en el párrafo 1 del Artículo 52 de la Carta se dispone que las actividades de los acuerdos y organismos regionales sean compatibles con los propósitos y principios de las Naciones Unidas. Esta disposición ha adquirido aún mayor validez en el mundo interdependiente de hoy, en que es inevitable que los acontecimientos de importancia de una región repercutan en otras. Por lo tanto, las actividades que se lleven a cabo en el marco de un regionalismo resurgente deberían complementar y no competir con las de las Naciones Unidas ni complicarlas. Para lograrlo hace falta una relación de trabajo entre las Naciones Unidas y los organismos regionales basada en la armonía y la confianza mutua. De lo contrario, la falta de coherencia y la fragmentación de las actividades de paz podrían actuar en desmedro de los propios mecanismos de paz. ${ }^{18}$

Boutros Boutros-Ghali dedicó todo el capítulo VII de su Agenda pour la Paix a tratar el tema de la cooperación entre las Naciones Unidas con los acuerdos y organismos regionales para el mantenimiento o el restablecimiento de la paz. Después de citar, en lo que se refiere a la América Latina, los casos de El Salvador y Nicaragua, como ejemplos de esa cooperación, ha dicho:

Ce qui est clair toutefois, c'est que les accords et organismes régionaux possèdent dans de nombreux cas un potentiel qui pourrait contribuer à l'accomplisement des fonctions examinées dans le présent rapport: diplimatie préventive, maintien de la paix, rétablissement de la paix et consolidation de la paix après les conflits. Aux termes de la charte, le Conseil de sécurité a - et continuera d'avoir - la responsabilité principale du maintien de la paix et de la sécurité internationales, mais l'action rétionale, par le biais de la décentralisation, de la délégation et de la coopération aux efforts de l'Organisation des Nations Unies, pourrait non seulement rendre plus légère la 
tâche du Conseil, mais contribuier ègalement a la création d'un sentiment plus fort de participation, de consensus et de démocratisation en ce qui concerne les affaires internationales. ${ }^{19}$

A este respecto es preciso citar, entre otros muchos ejemplos posibles, la resolución 49/57, adoptada en el 49 Periodo de Sesiones de la Asamblea General de las Naciones Unidas, en 1994 (Declaration sur le Renforcement de la Cooperation entre l'Organisation des Nationes Unies et les Accords ou Organismes regionaux dans le Domaine du Maintien de la Paix et de la Securité International), que al tratar del mantenimiento de la paz, se refiere a "la solución pacífica de diferendos y a la diplomacia preventiva".

\section{XI}

En América - especialmente en América Latina-, la prevención de los conflictos bélicos se fundó, durante largos años, en la existencia de un complejo y desarrollado sistema de solución pacífica de controversias, ${ }^{20}$ en iniciativas de diversa naturaleza, ${ }^{21} \mathrm{y}$ en limitadas y parciales decisiones respecto del desarme de armas convencionales y de destrucción masiva. $^{22}$

Pero fundamentalmente, se basó en el establecimiento, pionero y exitoso, de una zona libre de armas nucleares, establecida por el Tratado de

19 Boutros-Ghali, Boutros, op. cit., nota 5, pp. 11 y 12. Véase a este respecto: Cardona Llorens, Jorge, "La Cooperation entre las Naciones Unidas et les Accords et Organismes Regionaux pour le Reglament Pacifique des Affaires Relatives au Maintien de la Paix et de la Sécurité Internationales", en Boutros-Ghali, Boutros, Amirocum Discipulorumque Liber, Bruselas, Bruylant, vol. II, 1998; Píriz Ballón, Ramiro y Álvarez Goyoaga, Gustavo, “Algunos aspectos jurídicos que se plantean en relación a 'Un programa de paz', propuesto por el secretario general de las Naciones Unidas, Boutros Boutros-Ghali”, en varios autores, Liber Amicorum Eduardo Jiménez de Aréchaga, Montevideo, FCU, 1994, t. I, pp. 479-502.

20 El antiguo y completo Sistema Interamericano de Solución Pacífica de Controversias, que culminó con el Tratado Americano de Solución Pacífica o Pacto de Bogotá del 1948, incluyó ya en 1923 un Tratado para Evitar o Prevenir Conflictos entre los Estados Americanos (Pacto Gondra) y el Tratado General de Arbitraje Interamericano de 1929, que tuvo como objetivo hacer más eficiente el Pacto Gondra de 1923, y que se refiere especialmente a la forma de "prevenir" los conflictos.

21 Estas iniciativas se han ido individualizando y precisando con el transcurso del tiempo, especialmente en las dos últimas décadas, por medio de múltiples resoluciones de la Asamblea General, particularmente en lo que se refiere a las medidas de confianza.

22 Gros Espiell, Héctor, El sistema interamericano y el desarme, Washington, D. C., Comité Jurídico Interamericano, Curso de Derecho Internacional, XXV, 1998, pp. 195-230; Declaración de Ayacucho del 9 de diciembre de 1974; Reunión de México de agosto de 1978; Compromiso de Mendoza del 5 de septiembre de 1991 (Argentina, Brasil, Chile, Uruguay, Paraguay, Bolivia y Ecuador); Declaración de Cartagena de Indias del 4 de diciembre de 1991 (Bolivia, Colombia, Ecuador, Perú y Venezuela). 
Tlatelolco, ${ }^{23}$ que creó la zona libre de armas de la América Latina. Esta gran realización que sirvió de ejemplo y de modelo a la creación posterior de otras zonas análogas (Pacífico Sur, África, Asia del Sudeste, etcétera.) ${ }^{24}$ constituye sin duda la más original y exitosa contribución latinoamericana a la prevención de la guerra nuclear.

Una idea paralela es la creación de una zona de paz, concepto más amplio y totalizante que el de una zona libre de armas nucleares, que al incluir la proscripción de bases militares extranjeras y contener una serie de restricciones políticas y militares, aseguraría una prevención bélica amplia y comprensiva. ${ }^{25}$ Lamentablemente, pese a la existencia de

23 García Robles, Alfonso, "Mesures de Désarmement dans des Zones Particulieres: le Traité Visant l'Intrerdiction des Armes Nucleaires en Amerique Latine", Recueil des Cours, Academie de Droit International, vol. I, 1971; The Desnuclearization of Latin América, Carnegie Edowment for International Peace, 1967; La proscripción de armas nucleares en la América Latina, México, El Colegio Nacional, MCMLXXV; Gros Espiell, Héctor, En torno al Tratado de Tlatelolco y la Proscripción de las Armas Nucleares en América Latina, México, OPANAL, 1979; "Le Traité de Tlatelolco, un Texte Novateurs", Le Monde Diplomatique, París, Juillet, 1973; El Tratado de Tlatelolco y el derecho de los tratados, OPANAL, México, 1973; El Tratado de Tlatelolco; algunas consideraciones sobre aspectos específicos, México, OPANAL, 1970; "Las zonas libres de armas nucleares", Revista de Occidente, 3a. época, núms. 56, Madrid, 1979; "Los tratados del Canal de Panamá y las zonas libres de armas nucleares de la América Latina", Estudios en Honor de Adolfo Miaja de la Muela, tomo II, Madrid, 1979; "El Tratado de Tlatelolco", Boletín del Organismo Internacional de Energía Atómica, Viena, vol. 20, núm. 5, 1978; "Proscripçao de Armas Nucleares na América Latina", Revista de Dereito Nuclear, Río de Janeiro, núm. 1, 1979; El Tratado de Tlatelolco, Disarmament, Nueva York, United Nations, vol. II, núm. 2, 1980; "La no proliferación de armas nucleares en América Latina”, Boletín del Organismo Internacional de Energía Atómica, núm. 22, 3 y 4, Viena, 1980; “América Latina y el uso pacífico de la energía nuclear", Revista Mexicana de Política Exterior, México, núm. 50, 1996; Gros Espiell, Héctor, "Strengthening of Opanal: New Challengers for the Future", Nuclear -Weapon-Free Zones in the Next Century, United Nations, 1997; Bosco, Giorgio, Il Trattato di Tlatelolco, La Comunitá Internazionale, Roma, vol. XXI, núms. 1 y 2, 1974; Redick, John, Regional Nuclear Arms Control in Latin America, International Organization, primavera de 1975; Vigésimo Aniversario del Tratado de Tlatelolco, (19671987), México, OPANAL, 1988, con trabajos de Alfonso García Robles, Hans Blix, Julio César Carasales, Héctor Gros Espiell, Jorge Morelli Pando, Klauso Tornudd, Cristina Montaño, Daniel Lund, William Billinger, Michael Petrov, Djka Julius, Yosef Goldblal, Víctor Millán, David Sadleir, Pilar Armanet, Nigel Fyfe, Christopher Beeby, José R. Martírez Cobo y Antonio Stempel; “Armas nucleares, desarme y carrera armamentista, Homenaje a Alfonso García Robles", Jurídica, México, Universidad Iberoamericana, 1985, con estudios de Miguel Marín, Hubert Thierry, Modesto Seara Vázquez, Alicia Cabera, Saul Mandujano, Margarita González, Héctor Gros Espiell, Manfred Lachs, Sergio González Galves y Antonio Remiro Brotóns.

24 Gros Espiell, Héctor, Las zonas libres de armas nucleares, México; Boutros-Ghali, Boutros, Le Traité, de Pelindaba, Jeune Afrique, núm. 1840, abril de 1996; Mariño Menéndez, Fernando, Zonas libres de armas nucleares en el derecho internacional, Vitoria, 1985; Morey, Enrique Román, "El Tratado de Tlatelolco como ejemplo para la creación de nuevas zonas libres de armas nucleares", Revista Mexicana de Política Exterior, México, núm. 50, 1996.

25 Gros Espiell, Héctor, El concepto de zona de paz, Santiago de Chile, Comisión Sudamericana de Paz, 1988; id., "América del sur, zona de paz”, Revista Diplomacia, Santiago de Chile, núm. 61, 
múltiples iniciativas y a declaraciones de jefes de Estado (por ejemplo de los países del Mercosur (Argentina, Brasil, Paraguay y Uruguay), esta idea todavía no se ha concretado en América Latina en un tratado constitutivo.

\section{XII}

En Latinoamérica otras formas de medidas de prevención de conflictos bélicos, como las medidas de confianza, del tipo de la notificación de maniobras militares, observación de las mismas, información cruzada, etcétera, han sido aplicadas en las décadas de los ochenta y noventa, con positivos resultados. ${ }^{26}$ Deben citarse al respecto las declaraciones de Santiago (1995) y de El Salvador (1998) sobre Medidas de la Confianza y de la Seguridad, originadas en lo dispuesto en la Cumbre de Miami (1994) y una larga serie de resoluciones de la Asamblea General de la Organización de Estados Americanos, entre las que pueden citarse las AG/Res 1179 (XXII0/92), AG/Res. 1284 (XXIV0/94) y AG/Res. 1288 (XXIV0/94).

Las resoluciones de la Asamblea General de la OEA en los últimos años, en especial desde 1990 a 2000, sobre temas vinculados con el mantenimiento de la paz (seguridad hemisférica, armas pequeñas y ligeras, zonas libres de minas terrestres, armas químicas, zona libre de armas nucleares, transparencia en las adquisiciones de armas convencionales, fomento de confianza, no proliferación, gastos militares y registro de armas, y tráfico clandestino de armas), son muy abundantes y reiterativas.

1993; id., "Desarme regional, zonas libres de armas nucleares y zonas de paz”, Revista Española de Derecho Militar, Madrid, núm. 42, 1943; González Galves, Sergio, “América Latina como zona de paz”, Revista Mexicana de Política Exterior, México, núm. 50, 1990; Zona de paz en Centro América y el Caribe, Universidad para la paz, San José, Costa Rica, Flacso, 1989; Un análisis del tema de las zonas de paz, referido especialmente al Océano Pacífico, el Atlántico sur y el Mediterráneo, América Central, el Caribe y el sudeste asiático, Nepal y el Tibet, con olvido total de las iniciativas sudamericanas, puede verse en: MacAlister-Smith, Peter, "Zones of Peace", en Bernhart, R. (ed), Encyclopedia of Public International Law, 2000, vol. IV, pp. 1621-1633.

26 Para una nómina (inventario) de estas medidas de confianza en América Latina, véase el anexo II, "Sobre medidas de fomento de la confianza mutua", en Irela, Documento de Base, 1995. 
Hay que señalar, sin embargo, que esta proliferación resolutiva no se ha manifestado en una efectividad, en su proyección en la realidad de manera integral y en la forma que hubiera sido deseable.

\section{XIII}

En los casos de El Salvador, Honduras y Nicaragua, en los años pasados, medidas de confianza - como formas de prevención del posible resurgimiento de conflictos - para mantener la paz, aplicadas luego de la finalización de anteriores conflictos militares, han impedido el renacimiento de esos conflictos o el estallido de nuevos enfrentamientos. Han estado previstas en tratados, en acuerdos o en políticas regionales y mantienen hoy su importancia y efectividad. Permitieron llegar a la situación actual de tranquilidad y paz regional. Muchas veces estas medidas se han referido a conflictos bélicos internos con proyección o posible proyección internacional. ${ }^{27}$

Los acuerdos de paz entre Ecuador y Perú, firmados en Brasilia el 26 de octubre de 1998, ratificados por ambas partes, pusieron fin exitosamente a una intermitente situación de enfrentamiento bélico, que se remontaba al Protocolo de Río de Janeiro de 1942. Estos acuerdos, incluyeron fórmulas de prevención que se han demostrado eficaces en su aplicación, en una región en la que hoy reina la tranquilidad y la paz. Los seis acuerdos de Brasilia, firmados y aplicados por Ecuador y Perú, pueden considerarse en su conjunto como dirigidos a restablecer la paz, a mantenerla, a consolidarla y a prevenir el renacimiento de eventuales conflictos bélicos futuros. Pero hay que destacar entre ellas las Notas Reversales y el Acuerdo sobre Medidas de Confianza Mutua y Seguridad.

Situaciones recientes, potencialmente peligrosas, como las que se dieron, por ejemplo, entre Belice y Guatemala y entre Nicaragua y Costa Rica, no llegaron a plantear confrontaciones bélicas en razón de la utilización de activas formas de diplomacia preventiva, a veces de tipo bilateral, pero en la mayoría de los casos, apoyadas por acciones — de diferente naturaleza - emanadas de la Organización de Estados Americanos.

27 Nikken, Pedro, "El manejo del pasado y la cuestión de la imparcialidad en la solución de los conflictos armados en el Salvador y Guatemala", en varios autores, Liber Amicorum Héctor Fix-Zamudio, San José, Costa Rica, CIDH, 1998, vol. I, pp. 143-167; Buerghental, Thomas, "La Comisión de la Verdad para el Salvador", en Buerghental y Cançado Trindade, Estudios especializados en derechos humanos, San José, IIDH, 1996, t. I, pp. 11-62. 


\section{XIV}

La preservación de la democracia y la acción en caso de conflictos bélicos internos de posible grave proyección internacional, han permitido en América Latina concebir y emplear formas atípicas, pero necesarias, de prevención de conflictos. ${ }^{28}$ En esta prevención no puede olvidarse el peligro de la violación gravísima, masiva y reiterada de los derechos humanos así como los genocidios y la existencia de crímenes contra la humanidad, sus efectos desestabilizantes y sus proyecciones negativas para la seguridad internacional, con los consiguientes imperativos de acción.

El caso de Haití —entre otros ejemplos posibles, en que existió una cooperación de naturaleza muy especial ONU-OEA-, es particularmente destacable.

\section{XV}

En conclusión, la prevención de conflictos bélicos es un elemento esencial para que se pueda cumplir con el primer propósito de la Carta de las Naciones Unidas de mantener la paz y la seguridad internacionales.

La prevención de los conflictos bélicos constituye una forma, hoy imprescindible, para encarar la lucha por la proscripción de la amenaza o el uso de la fuerza (artículo 2, párrafo 4o. de la Carta de las Naciones Unidas). ${ }^{29}$

Esta prevención puede ejercerse mediante una gama muy amplia de posibles medidas, de diferente naturaleza y características. Supone actualmente la necesaria existencia de un sistema desarrollado de información y previsión.

La prevención puede cumplirse por medio de acciones inmediatas, contemporáneas con los hechos que pueden llevar al estallido bélico, pero

28 Reychler, L., "Democratic Peacebuilding and Conflicts Prevention”, International Law: Theory and Practice, Essays in Honour of Eric Suy, Edited by Karel Wellen, Nijhoff, The Hague, 1998; Gros Espiell, Héctor, "La democracia en el sistema interamericano", en Boutros-Ghali, Boutros, Amicorum Liber, Bruselas, Bruylant, 1997.

29 Los más recientes estudios sobre el uso de la fuerza, incluyen el tema de la prevención de conflictos, asunto que estaba casi siempre ausente en las obras dedicadas al tema del uso de la fuerza entre 1946 y los años noventa. Por ejemplo, el libro Gray, Christine, International Law and the Use of Force, Oxford-Nueva York, Oxford University Press, 2000, en el capítulo 6 (The U.N and the Use of Force), trata lo relativo a "Preventive Peacekeeping Forces" y "Cooperation with Regional Forces", pp. 198 y 199. 
no puede olvidarse en la prevención de conflictos el combate contra las causas o razones de éstas, sean de carácter político, militar, económico, social o cultural.

Los medios de solución pacífica de los conflictos constituyen conceptualmente una forma de prevención de los mismos.

Esto es especialmente importante con respecto a la acción de los tribunales internacionales, en particular de la Corte Internacional de Justicia y de los tribunales penales internacionales, como en los casos de los actuales de la ex-Yugoslavia y de Ruanda, y de la futura Corte Penal Internacional de Roma

Las fuerzas de paz, si actúan en los límites fijados por el derecho internacional, son un elemento de suma importancia en la prevención de los conflictos bélicos.

La prevención se complementa necesariamente con las medidas de mantenimiento y consolidación de la paz por adoptar, luego de finalizado un conflicto bélico.

Los conflictos bélicos internos pueden ser la causa u origen de conflictos bélicos internacionales. De aquí la necesidad de analizarlos y, en lo posible en ciertos casos, prevenirlos.

La diplomacia preventiva es actualmente un instrumento necesario para utilizar en la prevención de los conflictos bélicos.

La preservación de la democracia, la acción ante violaciones gravísimas, masivas y reiteradas de los derechos humanos y frente a genocidios y otros crímenes contra la humanidad, comienzan a constituir una forma de prevención de conflictos bélicos que se ha de acentuar y generalizar en el futuro.

En la prevención de los conflictos bélicos es esencial la coordinación armónica de las Naciones Unidas con la acción que puedan llegar a desarrollar los acuerdos u organismos regionales.

En el caso del sistema interamericano, esta acción conjunta de la OEA y de las Naciones Unidas, ante posibles conflictos bélicos futuros, ha dado excelentes resultados. Cabe esperar que esta situación continúe.

La prevención de conflictos en Latinoamérica ha conocido también formas de prevención de conflictos bélicos, basadas en acuerdos bilaterales o subregionales entre Estados de la región. 